\title{
Atorvastatin prevents Plasmodium falciparum cytoadherence and endothelial damage
}

\author{
Zacharie Taoufiq ${ }^{1,4^{*}}$, Paco Pino ${ }^{1,5}$, Nadine N'dilimabaka ${ }^{1}$, Issam Arrouss' ${ }^{1}$, Serge Assi ${ }^{1,6}$, Florent Soubrier ${ }^{2}$, \\ Angelita Rebollo ${ }^{1 \dagger}$, Dominique Mazier ${ }^{1,3^{*}+}$
}

\begin{abstract}
Background: The adhesion of Plasmodium falciparum parasitized red blood cell (PRBC) to human endothelial cells (EC) induces inflammatory processes, coagulation cascades, oxidative stress and apoptosis. These pathological processes are suspected to be responsible for the blood-brain-barrier and other organs' endothelial dysfunctions observed in fatal cases of malaria. Atorvastatin, a drug that belongs to the lowering cholesterol molecule family of statins, has been shown to ameliorate endothelial functions and is widely used in patients with cardiovascular disorders.
\end{abstract}

Methods: The effect of this compound on PRBC induced endothelial impairments was assessed using endothelial co-culture models.

Results: Atorvastatin pre-treatment of EC was found to reduce the expression of adhesion molecules and P. falciparum cytoadherence, to protect cells against PRBC-induced apoptosis and to enhance endothelial monolayer integrity during co-incubation with parasites.

Conclusions: These results might suggest a potential interest use of atorvastatin as a protective treatment to interfere with the pathophysiological cascades leading to severe malaria.

\section{Background}

Malaria remains a major threat to public health with $40 \%$ of the world population currently at risk. Every year, an estimated 500 million cases of clinical malaria and at least one million deaths are reported [1,2]. Fatal cases of malaria occur mainly in young children in Africa and consist of an acute neurological syndrome (cerebral malaria), either in isolation or concomitantly with multi-organ failure (pulmonary distress, acute renal failure) [3]. Anti-malarial drugs, such as quinine and artemisinin derivatives are administered intravenously as an emergency treatment. However, although these drugs effectively and rapidly clear parasites from the blood, $15 \%-20 \%$ of patients still die, probably as the result of impaired host responses $[4,5]$. Such a situation together with the difficulty in developing vaccines highlights the

\footnotetext{
* Correspondence: zacharie.taoufiq@wanadoo.fr; dominique.mazier@upmc.fr + Contributed equally

'INSERM, UMR S945, Université Pierre et Marie Curie-Paris 6, CHU-Pitié-

Salpêtrière, 91 bd de l'Hôpital, 75013 Paris, France

Full list of author information is available at the end of the article
}

urgent need of novel strategies for complement therapeutics.

The severity of Plasmodium falciparum infection depends largely on the ability of parasitized red blood cells (PRBC) to adhere on endothelial cells (EC) and sequester in the capillary network of vital organs (e.g. brain, lungs, kidneys, liver.) Moreover, activation of endothelial cells resulting from the adhesion of infected erythrocytes leads to an overexpression of different mediators, such as adhesion molecules ("hyperadhesion" phenomenon), pro-inflammatory cytokines, coagulation factors and contributes by itself to the pathology [6]. Among endothelial receptors, some are known to interact with PRBC, mainly via PfEMP1 (P. falciparum erythrocyte membrane protein 1 ), a highly polymorphic parasite ligand exported on the infected erythrocyte surface. A number of the cytoadherence receptors have been identified, such as ICAM-1, CD36, VCAM-1 or Pselectin [7]. It was previously shown that $P$. falciparum adhesion to human endothelial cells can specifically trigger proinflammatory gene expression [8-10], oxidative stress [11] and caspases activation [11], further leading

\section{Biomed Central}


to perturbation of the endothelial barrier integrity $[12,13]$. In addition PRBC adhesion to EC induces redox and rho-kinase dependent EC activation and apoptosis, which can be reversed respectively by the addition of anti-oxidants or fasudil rho-kinase inhibitor [12,14].

Atorvastatin is an oral drug that lowers the level of cholesterol in the blood. All statins, including atorvastatin, prevent the production of cholesterol by blocking the enzyme HMGCoA reductase. However, there is increasing evidence that statins may also exert effects beyond cholesterol lowering. Many of these cholesterol-independent or "pleiotropic" vascular effects of statins appear to involve restoring or improving the endothelial function through increasing the bioavailability of nitric oxide, promoting reendothelialization, reducing oxidative stress, and inhibiting inflammatory responses [15]. Thus, the endotheliumdependent effects of statins are thought to contribute to many of the beneficial effects of statin therapy in cardiovascular disease [16]. In addition to its lipids lowering effects, atorvastatin has been shown to promote nitric oxide (NO) production by decreasing caveolin-1 expression in EC, regardless of the level of extracellular LDL-cholesterol [17]. Statins retard the initiation of atherosclerosis formation through the improvement of NO bioavailability by both up-regulation of endothelial-nitric oxide synthase (eNOS) mRNA and decrease of superoxide anion $\mathrm{O}_{2}{ }^{-}$production in EC [18]. Statins modulate the adhesion cascade at multiple points by targeting both the endothelium and leukocytes and affect cell adhesion by inhibiting chemokine expression (MCP-1) in activated leukocytes and endothelial cells. There is also evidence that statins decrease ICAM-1 expression in stimulated EC and monocytes [19]. The effects of atorvastatin on mature EC are correlated with the activation of the anti-apoptotic Akt pathway, as determined by the phosphorylation of Akt and eNOS [20]. Therefore, activation of Akt represents a mechanism that can account for some of the beneficial side effects of statins.

Given the pleiotropic effects on endothelium of statins in general and atorvastatin in particular, atorvastatin was hypothesized to be useful as a protective drug against $P$. falciparum induced endothelial damages. Primary human lung endothelial cells (HLEC) were used here in co-culture with $P$. falciparum-infected erythrocytes Previous studies have already shown that PRBC adhesion triggers inflammation, oxidative stress and apoptosis within lung endothelial cells $[9,11,12,21,22]$. Moreover, acute respiratory distress as a complication of malaria infection is rare, but with a vey high rate of mortality $[3,23]$. The data presented in this manuscript show that, concomitantly with increased expression of Akt within HLEC, atorvastatin reduces adhesion of P. falciparum-infected erythrocytes, and as a consequence, prevents the endothelial damages induced by PRBC.

\section{Methods}

\section{Culture of human endothelial cell}

Primary endothelial cells were isolated from human lung (HLEC) after enzymatic digestion and selected using a continuous gradient and immunomagnetic purification technique, as described elsewhere [24]. Endothelial cells of ninth to twelfth passages derived from one batch were used for the experiments. Before use, cells were verified for their expression of ICAM-1, CD36, Von Willebrand factor, VCAM-1, CD31, E/P-selectin and CSA. HLEC were raised in M199 medium (Gibco), supplemented with $10 \mu \mathrm{g} / \mathrm{ml}$ of endothelial cell growth supplement (Upstate, NY) and $10 \%$ of fetal calf serum (Biowest), at $37^{\circ} \mathrm{C}$ with $5 \% \mathrm{CO}_{2}$, using 8 chamberLabtek (Nunc International, Napervil), $35 \mathrm{~mm}$ glass bottom dishes (MatTek corp.), 96-well plates (Costar), or Transwell insert supports (Corning LifeSciences).

\section{Plasmodium falciparum culture}

The P. falciparum 3D7 clone was used for these experiments. Infected erythrocytes were maintained in culture according to Trager and Jensen's technique in a suspension of erythrocytes in RPMI (Gibco) supplemented with $8.3 \mathrm{~g} / \mathrm{l}$ of Hepes, $2.1 \mathrm{~g} / \mathrm{l}$ of NaHCO, $0.1 \mathrm{mg} \cdot \mathrm{ml}^{-1}$ of gentamycin, $2 \mathrm{~g} / \mathrm{l}$ of dextrose, and $0.4 \%$ of Albumax II (Gibco, Invitrogen Corporation) [25]. The 3D7 clone was characterized for adhesion phenotype as previously described [25] and adheres to ICAM-1 and CD36. For each experiment, parasite cultures were enriched in mature forms by Plasmagel floating [26]. Briefly, erythrocytes were harvested from 5 to $10 \%$ parasitized cultures and centrifuged 5 minutes at $2000 \mathrm{rpm}$. Cells were resuspended in Plasmion ${ }^{\circledR}$ and incubated for 20 minutes at $37^{\circ} \mathrm{C}$. The upper fraction containing mature trophozoites and schizonts was collected and washed three times in RPM, before adequate adjustment of the hematocrit and parasitemia.

\section{Real time RT-PCR}

HLECs were treated with atorvastatin (Pfizer) for 24 hours, and total RNA was prepared using RNeasy mini kit (Qiagen). RT and quantitative PCR were performed as previously described [27]. Primers used were as follow: ICAM-1: forward 5'-GCAATGTGCAAGAAGA TAGCCA-3' reverse 5'-GGGCAAGACCTCAGGTC ATGT-3' VCAM-1: forward 5'-GAGTACG CAAACA CTTTATGTCAATGT-3', reverse 5'-CTCG TC CTTTCGGGACCG-3', P-selectin forward 5'-AGA CTCCC CACCAATGTGTGA-3', reverse 5'-CCACGAGTGTCA GAACAATCCA-3' CD36 forward 5'-TA 
ATGGCACA GATGCAGCCT-3', reverse 5'-ACAG CATAGATG GACCTGCAA-3' HPRT1 forward 5'-AA AGGACCC CACGAAGTGTT-3' reverse 5'-TCAAGG GCATATCCTACAACAA-3'.

\section{Plasmodium falciparum adhesion assay}

HLEC were raised in Labtek until confluence. Suspensions of mature pRBC were deposited onto cells and incubated for one hour at $37^{\circ} \mathrm{C}$ with gentle shaking every 10 minutes. After the incubation, the unbound pRBC were removed and the preparation was fixed for 30 minutes at room temperature with $2 \%$ glutaraldehyde, before staining with Giemsa. The number of parasites adhering to 700 HLEC was counted by direct observation with light microscope.

\section{Nucleosome release assay}

The proportion of apoptotic cells was assessed by measuring the intracytoplasmic release of mono- and oligonucleosomes as, using Cell Death Dtection ELISA ${ }^{\circledR}$ (Roche) previously described [11]. Briefly, HLEC were raised in 96-well plate (Costar) until confluence and exposed 24 hours to PRBC (hematocrit 5\% parasitemia $50 \%$ ) or RBC (hematocrit 5\%). After 5 PBS washing steps, endothelial cells were lysed and cytoplasms were analysed for their nucleosomes content.

\section{Endothelial barrier integrity assay}

Confluent endothelial monolayer were obtained by seeding 30,000 HLEC on Transwell permeable support (polyester, $3 \mu \mathrm{m}$ pores, $6.5 \mathrm{~mm}$ diameter) and raised in M199 medium supplemented as above during 36 hours. Endothelial monolayers were then exposed during 12 hours to PRBC suspensions at 1\% parasitemia and $0.5 \%$ of hematocrit. Transwell compartments were then washed three times and cell monolayers on Transwell inserts were transferred to a new plate containing PBS. Evans Blue $(0.5 \mathrm{mg} / \mathrm{mL})$ (ICN Biomedicals) was then added in the 'upper compartments'. After 5 min of incubation at $37^{\circ} \mathrm{C}$ and $5 \% \mathrm{CO}_{2}$, the 'under compartments' were collected for optic density analysis of diffused Evans Blue $(630 \mathrm{~nm})$ with a standard microplate reader (Bio-Tek ${ }^{\mathrm{TM}}$ EL311SX) [12].

\section{Western blot}

Cells $\left(1 \times 10^{6}\right)$ were lysed in Laemmli sample buffer and protein extracts were separated by SDS-PAGE, transferred to nitrocellulose, blocked ( $5 \%$ non-fat dry milk) in Tris-buffered saline (TBS: $20 \mathrm{mM}$ Tris- $\mathrm{HCl} \mathrm{pH} 7.5$, $150 \mathrm{mM} \mathrm{NaCl}$ ) plus $0.05 \%$ Tween-20 and incubated with the primary antibody over night at $4{ }^{\circ} \mathrm{C}$ in TBS$0.5 \%$ non-fat dry milk. The membrane was washed and incubated with PO-conjugated secondary antibody for two hours at room temperature. Secondary antibodies on western blot membranes were revealed using the ECL system.

\section{Immunofluorescence and confocal microscopy}

HLEC were raised in $35 \mathrm{~mm}$ glass bottom dishes, before fixation with $1 \%$ paraformaldehyde for $5 \mathrm{~min}$, permeabilized and then incubated with polyclonal anti-Akt antibody (Cell Signaling) for $2 \mathrm{~h}$ in PBS 3\% BSA at room temperature. FITC-secondary antibody was added and incubated for $1 \mathrm{~h}$ at room temperature. After several washing steps, samples were incubated with methanol at $-20^{\circ} \mathrm{C}$ for 10 min, mounted with Vectashield medium and analysed by confocal microscopy.

\section{Statistical analysis}

Differences between groups were analysed for statistical significance using the Games-Howell post-hoc (SPSS software). A $p$ value at least less than 0.05 was considered significant.

\section{Results and discussion}

Atorvastatin prevents $P$. falciparum-induced endothelial adhesion molecules upregulation

In addition to their anti-cholesterol function, statins are also involved in expression regulation of some adhesion molecules in lymphocytes, monocytes and endothelial cells. The role of statin atorvastatin in the control of expression of CD36, ICAM-1, VCAM-1 and P-Selectin, four adhesion molecules that are involved in tethering, rolling and adhesion processes, was analysed in a coculture of endothelial cells and P. falciparum parasitized red blood cells (PRBC). Atorvastatin doses, ranging from 0.01 to 1 microM, showed no toxic effect on human lung endothelial cells (HLEC) in vitro, doses ranging from 1.5 microM to 5 microM showed low toxic effects, whereas atorvastatin doses above 6 microM showed mitochondrial toxic effects on HLEC (MTT mitochondrial-based cell viability assay). HLEC were pre-treated 24 hours with 1 microM of atorvastatin before being exposed to PRBC or control RBC during four hours. Total RNA of EC was extracted and adhesion molecules expression was assessed by qPCR. On the one hand, our data show that PRBC increase the expression of endothelial cell (EC) adhesion molecule ICAM-1 and P-selectin. Indeed, PRBC cytoadherence was previously shown to increase cytoadherence itself through increase of EC adhesion molecules expression ('hyperadhesion') [28], contributing to the microcirculation perturbation in severe malaria pathology. More importantly, the data clearly showed that the P. falciparum-induced increase of ICAM-1 and P-Selectin expression is suppressed by atorvastatin pre-treatment (Figure 1). 


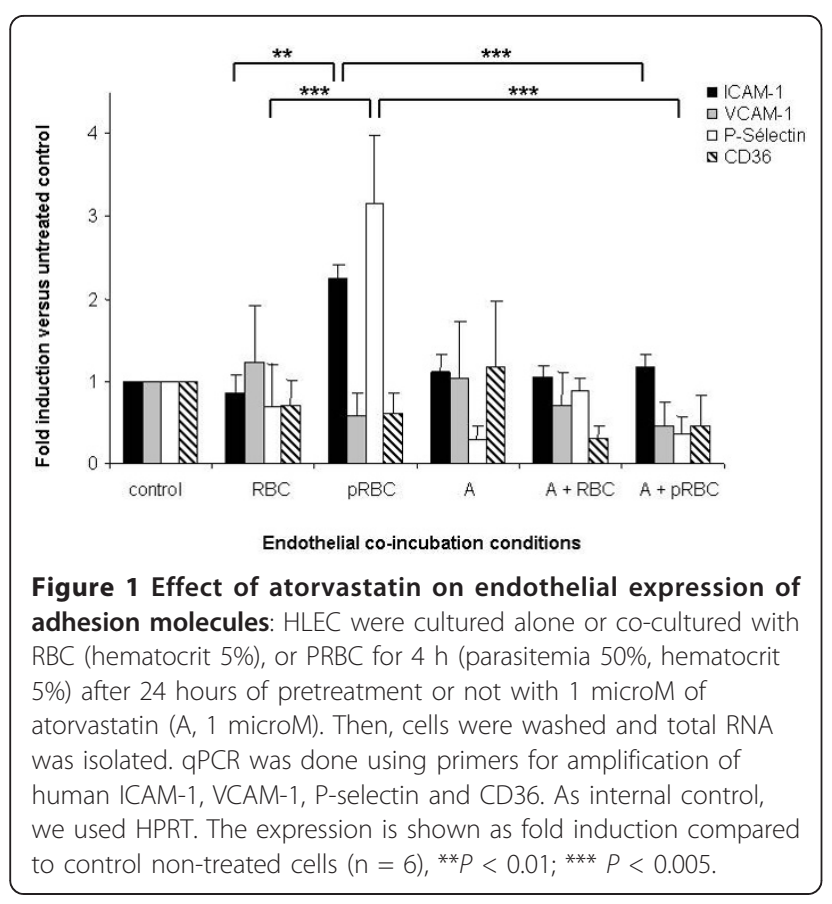

Atorvastatin decreases $P$. falciparum cytoadherence and $P$. falciparum-induced endothelial apoptosis

Given the effect of atorvastatin endothelial treatment on the expression of adhesion molecules, its effect on PRBC ability to adhere on endothelial cells was analysed (Figure 2). HLEC were pre-incubated 24 hours with various doses of atorvastatin $(0.01,0.025,0.05,0.1,0.5$, 1 microM), before being exposed to PRBC ad

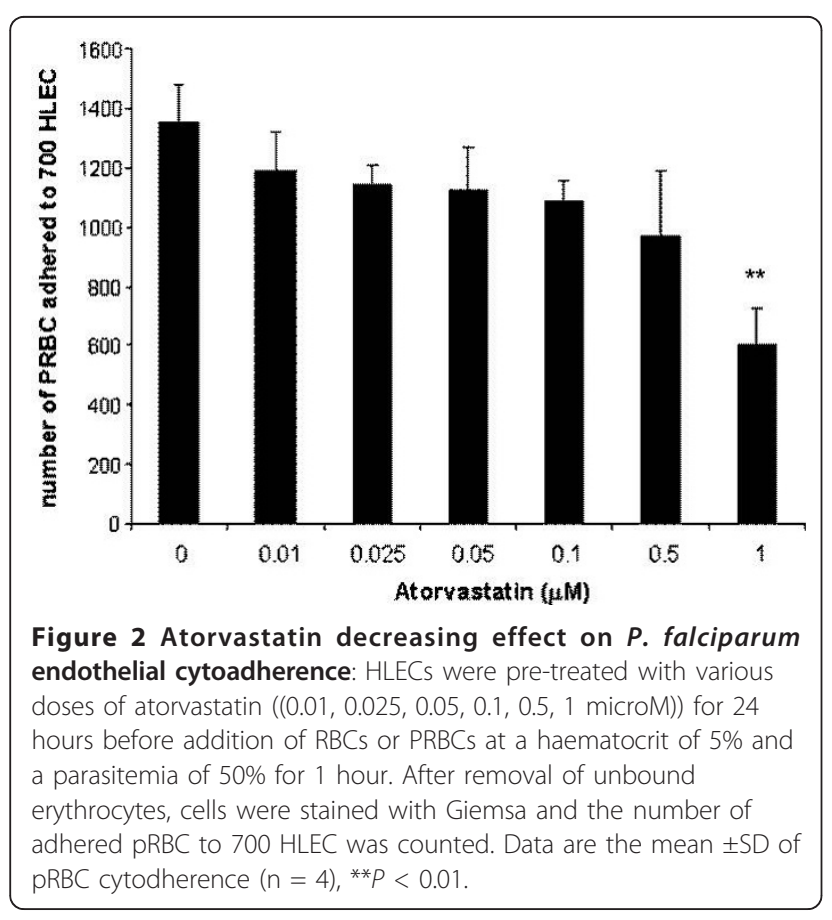

cytoadherence assay. The data showed that PRBC cytoadherence is significantly decreased by doses that are higher than 0.5 microM of atorvastatin. The cytoadherence decreased to $44 \% \pm 9$, for EC pre-incubated with 1 microM of atorvastatin compared to untreated controls. Atorvastatin is thus capable of interfering efficiently with the adhesion of $P$. falciparum infected erythrocytes on endothelial cells.

PRBC adhesion was previously shown to specifically trigger endothelial apoptosis [9]. The effect of atorvastatin (0.01-1 microM) on endothelial apoptosis was tested in the co-culture model. HLEC were pre-treated 24 hours with atorvastatin before being exposed for four hours to PRBC or control uninfected RBC. Endothelial apoptosis was then quantitatively assessed using a method to determine HLEC intracytoplasmic content of nucleosomes, a well known late apoptosis marker (Figure 3). The data showed decreasing endothelial apoptosis correlated with increasing doses of atorvastatin pre-treatment. PRBC-induced apoptosis was found completely abolished with doses higher than 0.05 microM.

\section{Atorvastatin protects endothelial barrier integrity from $P$. falciparum-induced impairments}

PRBC were previously observed to trigger specific signaling pathway that leads to endothelial barrier permeabilization [12]. Given the effects of atorvastatin pre-treatments on endothelial apoptosis, atorvastatin was tested whether it could have also beneficial properties on endothelial barrier integrity. HLEC were pre-treated with 1 microM of atorvastatin for $24 \mathrm{~h}$ and then with PRBC for 4 hours.

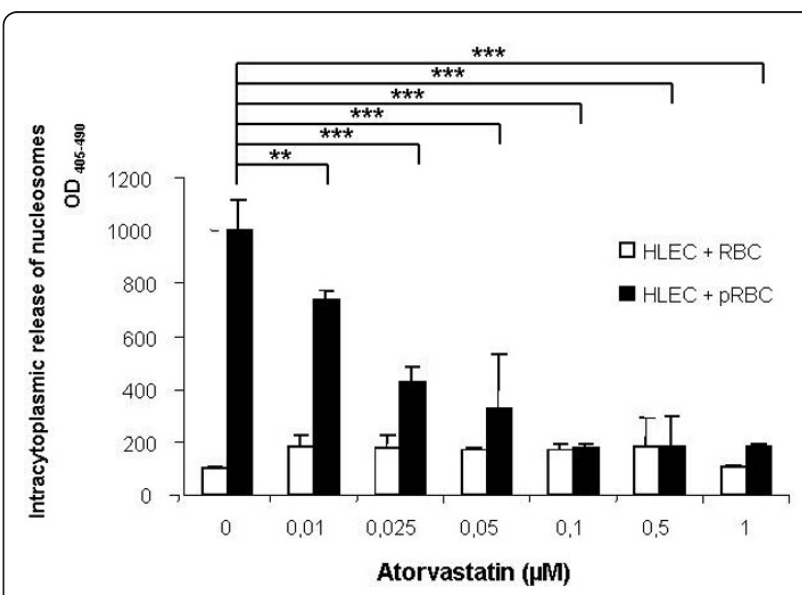

Figure 3 Effect of atorvastatin on P. falciparum-induced endothelial apoptosis: HLEC cells were pre-treated with or without different concentrations of atorvastatin $((0.01,0.025,0.05,0.1,0.5,1$ microM)) for $24 \mathrm{~h}$ and then co-cultured 4 hours with PRBC

(hematocrit 5\% parasitemia 50\%) or RBC (hematocrit 5\%).

Intracytoplasmic content of nucleosomes was assessed $(n=4)$, **P $<0.01$; ${ }^{* *} P<0.005$. 
After washing of endothelial monolayer, the cell barrier integrity was assessed by the standard Evans blue extrusion method. Figure 4 shows that co-culture of PRBC with endothelial cells induces endothelial barrier permeability, compared to unexposed control cells or RBC exposed cells. Pre-treatment of endothelial cells with atorvastatin 1 microM could strongly decrease PRBC-inducced barrier permeabilization. This result suggests that atorvastatin is also very efficient to protect endothelial barrier integrity from P. falciparum-induced impairments. However, it should be noted that the relevance of these quantitative data must be restricted to the detection method of standard Evans blue extrusion.

\section{Atorvastatin increases Akt expression in endothelial cells exposed to $P$. falciparum}

Among the pleiotropic effects of statins, their role in the activation of cell survival protein kinase B or Akt signaling pathway is well established [29,30]. Given the protective effect of atorvastatin against cell death in our endothelialPRBC co-culture model, the effect of atorvastatin treatment on endothelial Akt expression was analysed. HLEC were treated 24 hours with $0.05,1$ or 5 microM of atorvastatin. Figure 5A shows Western blot data and its respective quantitative analysis by densitometry imaging. It shows that pre-treatment of increasing doses of atorvastatin up-regulates Akt expression in HLEC. Similarly, Akt expression was up-regulated in co-cultures of HLEC exposed to $\mathrm{RBC}$ or PRBC. The effect of atorvastatin on the endothelial up-regulation of Akt expression was confirmed by confocal microscopy imaging analyses (Figure $5 \mathrm{~B}$ ). These data suggest that atorvastatin increases the Akt expression within endothelial cells.

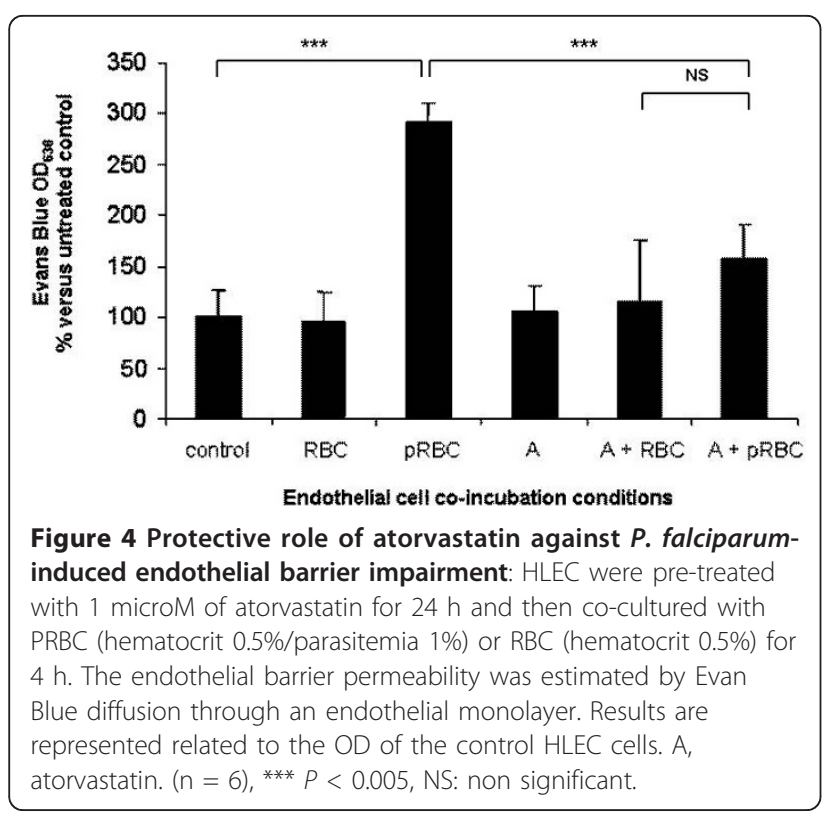

The data presented here demonstrate that atorvastatin can be used to reduce the cytoadherence of $P$. falciparum on endothelial cells, which is one of key events, along with the inflammatory burst, involved in the pathogenesis of human severe malaria cases. Atorvastatin can decrease the expression of adhesion molecules and also prevents the PRBC-induced overexpression of adhesion molecules. Moreover, atorvastatin shows an ability to strongly protect endothelial cell against $P$. falciparum-induced collateral damages, cell apoptosis and endothelial barrier permeabilization.

The observed cytoprotection effect on endothelial cells may mainly be due to the decrease of PRBC adhesion through the down-regulation of adhesion molecules, as PRBC contact was previously shown to specifically trigger pro-inflammatory and pro-apoptotic signaling cascades [9]. Moreover, atorvastatin stimulates Akt expression within HLEC, which is the major actor of anti-apoptotic and endothelial cell survival pathways [29]. Endothelial protection of atorvastatin appears then to be 'pleiotropic' via anti-adhesive, anti-inflammatory and anti-apoptotic synergistic effects. Indeed, PRBC cytoadherence is known to activate many deleterious cascades such as oxidative stress, via mitochondrial ROS (radical oxygen species) production, rho-kinase and NF-kappa B-dependent signaling to induce 'hyperadhesion' phenomenon, endothelial activation and apoptosis $[8,11,12,14,28,31]$. When phosphorylated, Akt inactivates pro-apoptotic elements such as Bad, preventing mitochondrial ROS release, and caspase 9 [32]. Statins can also increase the bioavailability and the physiological production of endothelial nitric oxide (NO) via Akt phosphorylation and downstream effector endothelial NO-synthase (eNOS), and via the stabilization of eNOS mRNA [30,33]. NO has many crucial functions in vascular endothelium particularly in maintaining permanently anti-inflammatory and antiadhesive endothelium homeostasis. NO-donors have currently been under investigation in cerebral malaria adjunctive emergency treatments, the efficiency of which remains controversial [34]. Indeed, the deleterious scavenging of NO by ROS has not been considered with antioxidant co-treatment yet. As regard their molecular 'pleiotropic' effects, particularly with the antioxidant and the NO bioavailability promotion, statins may then be highly relevant to 'shield' the endothelium against both PRBC induced impairments and uncontrolled host responses in severe malaria cases.

There is increasing interest of using statins as complementary therapeutic to anti-plasmodial molecules. However, these studies with statins were performed on infected erythrocytes only and have shown interestingly to have a direct inhibitory effect on $P$. falciparum growth in vitro, using high doses ranging from 120 to 240 microM [35], from 5 to 40 microM [36] or from 


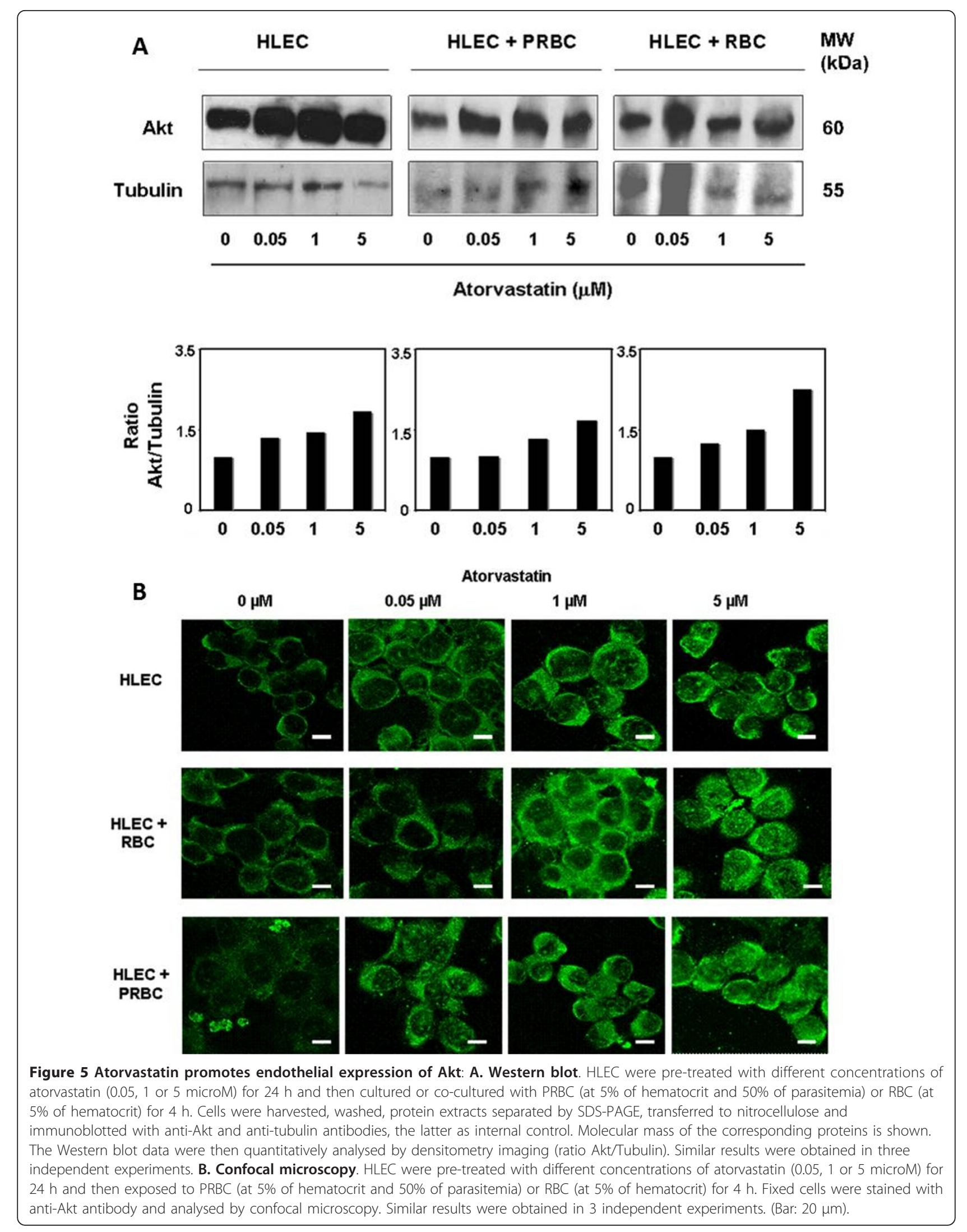


2.5 to 10.8 microM [37]. Atorvastatin was shown to be 10 times more active against $P$. falciparum compared to mevastatin, simvastatin, lovastatin, fluvastatin or pravastatin [36], suggesting that atorvastatin is the best candidate among statins for therapy prospects in malaria treatment. Simvastatin was also used in mouse model of cerebral malaria (C57BL/6 mice infected with Plasmodium berghei), but failed to decrease significantly parasitemia or to prevent death [38,39]. Atorvastatin may have a beneficial effect on mice survival, only when administered in combination with artesunate [40]. Indeed, atorvastatin was recently shown to reduce the IC50 of the anti-plasmodial activity of quinine, mefloquine and dihydroartemisinin [41-43]. However, the pathogenesis of cerebral malaria in murine model relies solely on the inflammatory response unlike the pathogenesis of human cerebral malaria. Indeed, the cytoadherence phenomenon does not exist in P. berghei mice infections $[44,45]$. Atorvastatin was used in pre-treatment to specifically evaluate the potential effects on endothelial cells. Endothelial damage is almost universal in severe malaria pathogenesis and statins have proved their efficiency in clinical use for 20 years against cardiovascular diseases [29]. Interestingly, significant endothelial protection effects were obtained with doses as low as 100 nanoM, 500 nanoM or 1 microM.

Although the 'pleiotropic' effects of statins are well documented, the precise molecular mechanism by which the PI3K/Akt/eNOS pathway is activated remains unknown $[29,46]$. Since the cholesterol lowering property of statins by itself ameliorates the endothelial function, statins beneficial effects were for long attributed to the inhibition of HMG-CoA reductase and the cholesterol synthesis pathway. However, the endothelial function improvement was shown to occur earlier than cholesterol lowering and independently from the cholesterol rate in blood serum $[47,48]$. Statins are inhibiting the synthesis of mevalonate, the second biochemical reaction in the cholesterol synthesis pathway. Mevalonate is also an important precursor of isoprenoid intermediates such as farnesyl pyrophosphate (FPP) or geranylgeranyl pyrophosphate (GGPP). Isoprenoids serve as cell surface inner membrane-anchoring molecules to functionalize RhoGTPases, such as Rho (GGPP) and Ras (FPP) [49,50], key signaling molecules, after geranylation and farnesylation post-translational modifications. Rho-GTPases act as molecular switches (active and inactive when bound to GTP and GDP, respectively), and are the first intermediates of the intracellular signaling engagement of these receptors with the downstream effector Rho-kinase. We have previously demonstrated that $P$. falciparum-induced endothelial collateral damages are precisely dependent on Rho-kinase signaling [12]. Rho-kinase pathway by many aspects is antagonist to PI3K/Akt/eNOS endothelial cell survival pathway [46]. In fact, strong endothelial protective effects, obtained with atorvastatin against $P$. falciparum, may likely be related to both rho-kinase pathway inhibition and Akt cell survival pathway promotion. However, it was previously reported that the rho-kinase inhibition by fasudil could not decrease significantly the number of parasites and $P$. falciparum cytoadherence $[12,51]$.

\section{Conclusions}

In conclusion, data from this report demonstrate that HMG-CoA reductase inhibitor atorvastatin prevents $P$. faciparum cytoadherence and confers the ability to the endothelium to resist against consequent cellular damages. In conclusion, this study suggests that atorvastatin may be a good candidate for further studies or clinical trials on the use of statins in malaria treatment. Moreover, the safety of statins on malaria treatment needs to be addressed, since these molecules were not originally designed for these therapeutic approaches.

List of abbreviations

EC: endothelial cell; RBC: red blood cells; PRBC: parasitized red blood cells; HLEC: human lung endothelial cells.

\section{Acknowledgements and funding}

We are grateful to David Walliker for the gift of the 3D7 clone, We also thank Kabongo Muanza for HLEC isolation, Maurel Tefit for technical assistance, and loannis Vouldoukis for critical manuscript discussions. Zacharie Taoufiq was financially supported by the Ministère de l'Education Nationale, de la Recherche et de la Technologie.and by la Fondation pour la Recherche Médicale (FRM).

\section{Author details}

${ }^{1}$ INSERM, UMR 5945, Université Pierre et Marie Curie-Paris 6, CHU-PitiéSalpêtrière, 91 bd de I'Hôpital, 75013 Paris, France. ${ }^{2}$ INSERM, UMR S525, Université Pierre et Marie Curie-Paris 6, Paris, France. ${ }^{3}$ AP-HP, Groupe hospitalier Pitié-Salpêtrière, Service Parasitologie-Mycologie, Paris, France. ${ }^{4}$ Okinawa Institute of Science and Technology, Onna, Okinawa, Japan. ${ }^{5}$ Department of Microbiology and Molecular Medicine, University of Geneva, Geneva, Switzerland. ${ }^{6}$ Institut Pierre Richet/Institut National de la Sante Publique, Abidjan, Ivory Coast.

\section{Authors' contributions}

ZT contributed to write the manuscript, to design and to conduct the experiments. PP contributed to design the experiments. NN, IA and SA contributed to perform some experiments. FS made substantial constructive advice in the initial design of the project. AR and DM contributed to write the manuscript and to design the experiments. All authors have read and approved the final version of the manuscript.

\section{Competing interests}

The authors declare that they have no competing interests.

Received: 30 November 2010 Accepted: 28 February 2011 Published: 28 February 2011

\section{References}

1. Snow RW, Guerra CA, Noor AM, Myint HY, Hay SI: The global distribution of clinical episodes of Plasmodium falciparum malaria. Nature 2005, 434:214-217.

2. UNICEF/WHO: World Malaria Report 2009. 2009 [http://www.whoint/ malaria/world_malaria_report_2009]. 
3. Marsh K, Forster D, Waruiru C, Mwangi I, Winstanley M, Marsh V, Newton C, Winstanley P, Warn P, Peshu N, Pasvol G, Snow R: Indicators of lifethreatening malaria in African children. N Engl J Med 1995, 332:1399-1404.

4. Dondorp A, Nosten F, Stepniewska K, Day N, White N: Artesunate versus quinine for treatment of severe falciparum malaria: a randomised trial. Lancet 2005, 366:717-725.

5. Idro R, Jenkins NE, Newton CR: Pathogenesis, clinical features, and neurological outcome of cerebral malaria. Lancet Neurol 2005, 4:827-840.

6. van der Heyde HC, Nolan J, Combes V, Gramaglia I, Grau GE: A unified hypothesis for the genesis of cerebral malaria: sequestration, inflammation and hemostasis leading to microcirculatory dysfunction. Trends Parasitol 2006, 22:503-508

7. Ho M, White NJ: Molecular mechanisms of cytoadherence in malaria. Am J Physiol 1999, 276:1231-1242

8. Jenkins N, Wu Y, Chakravorty S, Kai O, Marsh K, Craig A: Plasmodium falciparum intercellular adhesion molecule-1-based cytoadherencerelated signaling in human endothelial cells. J Infect Dis 2007, 196:321-327.

9. Pino P, Vouldoukis I, Kolb JP, Mahmoudi N, Desportes-Livage I, Bricaire F, Danis M, Dugas B, Mazier D: Plasmodium falciparum-infected erythrocyte adhesion induces caspase activation and apoptosis in human endothelial cells. J Infect Dis 2003, 187:1283-1290.

10. Tripathi AK, Sha W, Shulaev V, Stins MF, Sullivan DJ Jr: Plasmodium falciparum-infected erythrocytes induce NF-kappaB regulated inflammatory pathways in human cerebral endothelium. Blood 2009, 114:4243-4252.

11. Pino P, Vouldoukis I, Dugas N, Hassani-Loppion G, Dugas B, Mazier D: Redox-dependent apoptosis in human endothelial cells after adhesion of Plasmodium falciparum-infected erythrocytes. Ann N Y Acad Sci 2003, 1010:582-586

12. Taoufiq Z, Gay F, Balvanyos J, Ciceron L, Tefit M, Lechat P, Mazier D: Rho kinase inhibition in severe malaria: thwarting parasite-induced collateral damage to endothelia. J Infect Dis 2008, 197:1062-1073.

13. Tripathi AK, Sullivan DJ, Stins MF: Plasmodium falciparum-infected erythrocytes decrease the integrity of human blood-brain barrier endothelial cell monolayers. J Infect Dis 2007, 195:942-950.

14. Taoufiq Z, Pino P, Dugas N, Conti M, Tefit M, Mazier D, Vouldoukis I: Transient supplementation of superoxide dismutase protects endothelial cells against Plasmodium falciparum-induced oxidative stress. $\mathrm{Mol}$ Biochem Parasitol 2006, 150:166-173.

15. Laufs U: Beyond lipid-lowering: effects of statins on endothelial nitric oxide. Eur J Clin Pharmacol 2003, 58:719-731

16. Rosenson RS: Pluripotential mechanisms of cardioprotection with HMGCoA reductase inhibitor therapy. Am J Cardiovasc Drugs 2001, 1:411-420.

17. Brouet A, Sonveaux P, Dessy C, Moniotte S, Balligand JL, Feron O: Hsp90 and caveolin are key targets for the proangiogenic nitric oxidemediated effects of statins. Circ Res 2001, 89:866-873.

18. Sumi D, Hayashi T, Thakur NK, Jayachandran M, Asai $Y$, Kano H, Matsui $H$, Iguchi A: A HMG-CoA reductase inhibitor possesses a potent antiatherosclerotic effect other than serum lipid lowering effects-the relevance of endothelial nitric oxide synthase and superoxide anion scavenging action. Atherosclerosis 2001, 155:347-357.

19. Romano M, Diomede L, Sironi M, Massimiliano L, Sottocorno M, Polentarutti N, Guglielmotti A, Albani D, Bruno A, Fruscella P, Salmona M, Vecchi A, Pinza M, Mantovani M: Inhibition of monocyte chemotactic protein-1 synthesis by statins. Lab Invest 2000, 80:1095-1100.

20. Urbich C, Dernbach E, Zeiher AM, Dimmeler S: Double-edged role of statins in angiogenesis signaling. Circ Res 2002, 90:737-744.

21. Siau A, Toure FS, Ouwe-Missi-Oukem-Boyer O, Ciceron L, Mahmoudi N, Vaquero C, Froissard P, Bisvigou U, Bisser S, Coppee JY, Bischoff E, David PH, Mazier D: Whole-transcriptome analysis of Plasmodium falciparum field isolates: identification of new pathogenicity factors. J Infect Dis 2007, 196:1603-1612

22. Zang-Edou ES, Bisvigou U, Taoufiq Z, Lekoulou F, Lekana-Douki JB, Traore Y, Mazier D, Toure-Ndouo FS: Inhibition of Plasmodium falciparum field isolates-mediated endothelial cell apoptosis by Fasudil: therapeutic implications for severe malaria. PLOS One 2010, 5:e13221.

23. Mishra SK, Mohanty S, Mohanty A, Das BS: Management of severe and complicated malaria. J Postgrad Med 2006, 52:281-287.

24. Muanza K, Gay F, Behr C, Scherf A: Primary culture of human lung microvessel endothelial cells: a useful in vitro model for studying
Plasmodium falciparum-infected erythrocyte cytoadherence. Res Immunol 1996, 147:149-163.

25. Trager W: Cultivation of malaria parasites. Methods Cell Biol 1994, 45:7-26.

26. Goodyer ID, Johnson J, Eisenthal R, Hayes DJ: Purification of mature-stage Plasmodium falciparum by gelatine flotation. Ann Trop Med Parasitol 1994, 88:209-211.

27. Brun M, Bourdoulous S, Couraud PO, Elion J, Krishnamoorthy R, Lapoumeroulie C: Hydroxyurea downregulates endothelin-1 gene expression and upregulates ICAM-1 gene expression in cultured human endothelial cells. Pharmacogenomics J 2003, 3:215-226.

28. Tripathi AK, Sullivan DJ, Stins MF: Plasmodium falciparum-infected erythrocytes increase intercellular adhesion molecule 1 expression on brain endothelium through NF-kappaB. Infect Immun 2006, 74:3262-3270.

29. Mason JC: Statins and their role in vascular protection. Clin Sci (Lond) 2003, 105:251-266.

30. Wolfrum S, Jensen KS, Liao JK: Endothelium-dependent effects of statins. Arterioscler Thromb Vasc Biol 2003, 23:729-736.

31. Schofield L, Novakovic S, Gerold P, Schwarz RT, McConville MJ, Tachado SD: Glycosylphosphatidylinositol toxin of Plasmodium up-regulates intercellular adhesion molecule-1, vascular cell adhesion molecule-1, and E-selectin expression in vascular endothelial cells and increases leukocyte and parasite cytoadherence via tyrosine kinase-dependent signal transduction. J Immunol 1996, 156:1886-1896.

32. Datta SR, Dudek H, Tao X, Masters S, Fu H, Gotoh Y, Greenberg ME: Akt phosphorylation of BAD couples survival signals to the cell-intrinsic death machinery. Cell 1997, 91:231-241.

33. Endres M, Laufs U, Liao JK, Moskowitz MA: Targeting eNOS for stroke protection. Trends Neurosci 2004, 27:283-289.

34. Sobolewski P, Gramaglia I, Frangos J, Intaglietta M, van der Heyde HC: Nitric oxide bioavailability in malaria. Trends Parasitol 2005, 21(9):415-422.

35. Couto AS, Kimura EA, Peres VJ, Uhrig ML, Katzin AM: Active isoprenoid pathway in the intra-erythrocytic stages of Plasmodium falciparum: presence of dolichols of 11 and 12 isoprene units. Biochem J 1999, 341:629-637.

36. Pradines B, Torrentino-Madamet M, Fontaine A, Henry M, Baret E, Mosnier J, Briolant S, Fusai T, Rogier C: Atorvastatin is 10 -fold more active in vitro than other statins against Plasmodium falciparum. Antimicrob Agents Chemother 2007, 51:2654-2655.

37. Parquet $\mathrm{V}$, Briolant S, Torrentino-Madamet M, Henry M, Almeras L, Amalvict $R$, Baret $E$, Fusai $T$, Rogier $C$, Pradines $B$ : Atorvastatin is a promising partner for antimalarial drugs in treatment of Plasmodium falciparum malaria. Antimicrob Agents Chemother 2009, 53:2248-2252.

38. Helmers AJ, Gowda DC, Kain KC, Liles WC: Statins fail to improve outcome in experimental cerebral malaria and potentiate Toll-like receptormediated cytokine production by murine macrophages. Am J Trop Med Hyg 2009, 81:631-637.

39. Kobbe R, Schreiber N, May J, Jacobs T: Simvastatin treatment shows no effect on the incidence of cerebral malaria or parasitemia during experimental malaria. Antimicrob Agents Chemother 2008, 52:1583-1584.

40. Bienvenu AL, Picot S: Statins alone are ineffective in cerebral malaria but potentiate artesunate. Antimicrob Agents Chemother 2008, 52:4203-4204.

41. Parquet V, Henry M, Wurtz N, Dormoi J, Briolant S, Gil M, Baret E, Amalvict R, Rogier C, Pradines B: Atorvastatin as a potential anti-malarial drug: in vitro synergy in combinational therapy with quinine against Plasmodium falciparum. Malar J 2010, 9:139.

42. Savini H, Souraud JB, Briolant S, Baret E, Amalvict R, Rogier C, Pradines B: Atorvastatin as a potential antimalarial drug: in vitro synergy in combinational therapy with dihydroartemisinin. Antimicrob Agents Chemother 2010, 54:966-967.

43. Wurtz N, Briolant S, Gil M, Parquet V, Henry M, Baret E, Amalvict R, Almeras $L$, Rogier $C$, Pradines B: Synergy of mefloquine activity with atorvastatin, but not chloroquine and monodesethylamodiaquine, and association with the pfmdr1 gene. J Antimicrob Chemother 2010, 65:1387-1394.

44. Lackner P, Beer R, Helbok R, Broessner G, Engelhardt K, Brenneis C, Schmutzhard E, Pfaller K: Scanning electron microscopy of the neuropathology of murine cerebral malaria. Malar J 2006, 5:116.

45. White NJ, Turner GD, Medana IM, Dondorp AM, Day NP: The murine cerebral malaria phenomenon. Trends Parasitol 2009, 26:11-15.

46. Zhou Q, Liao JK: Statins and cardiovascular diseases: from cholesterol lowering to pleiotropy. Curr Pharm Des 2009, 15:467-478. 
47. MRC/BHF: MRC/BHF Heart Protection Study of cholesterol lowering with simvastatin in 20,536 high-risk individuals: a randomised placebocontrolled trial. Lancet 2002, 360:7-22.

48. O'Driscoll G, Green D, Taylor RR: Simvastatin, an HMG-coenzyme A reductase inhibitor, improves endothelial function within 1 month. Circulation 1997, 95:1126-1131.

49. Goldstein JL, Brown MS: Regulation of the mevalonate pathway. Nature 1990, 343:425-430.

50. Van Aelst L, D'Souza-Schorey C: Rho GTPases and signaling networks. Genes Dev 1997, 11:2295-2322.

51. Waknine-Grinberg JH, McQuillan JA, Hunt N, Ginsburg H, Golenser J: Modulation of cerebral malaria by fasudil and other immune-modifying compounds. Exp Parasitol 2010, 125:141-146.

doi:10.1186/1475-2875-10-52

Cite this article as: Taoufiq et al:: Atorvastatin prevents Plasmodium

falciparum cytoadherence and endothelial damage. Malaria Journal 2011 10:52.

\section{Submit your next manuscript to BioMed Central} and take full advantage of:

- Convenient online submission

- Thorough peer review

- No space constraints or color figure charges

- Immediate publication on acceptance

- Inclusion in PubMed, CAS, Scopus and Google Scholar

- Research which is freely available for redistribution

Submit your manuscript at www.biomedcentral.com/submit 